BULL. AUSTRAL. MATH. SOC.

\title{
NORMAL AND SUBNORMAL SUBGROUPS \\ IN THE GROUP OF UNITS OF GROUP RINGS
}

\author{
JAIRO ZaCARIAS GONCALVES
}

\begin{abstract}
Let $K G$ be the group ring of the group $G$ over the infinite field $K$, and let $U(K G)$ be its group of units. If $G$ is torsion, we obtain necessary and sufficient conditions for a finite subgroup $H$ of $G$ to be either normal or subnormal in $U(K G)$. Actually, if $H$ is subnormal in $U(K G)$, we can handle not only the case $H$ finite, but the precise assumptions depend on the characteristic of $K$.
\end{abstract}

\section{Introduction}

Let $R G$ be the group ring of the group $G$ over an integral domain $R$, and let $U(R G)$ be its group of units. When $R=K$, an infinite field of characteristic $p>0$, and $G$ is a torsion group we show that finite normal and subnormal subgroups of $U(K G)$ are central or "almost" central. This has a strong resemblance to the case in which $G$ is finite, and is in the same Iine as Pearson [6], and Pearson and Taylor [7].

If $R=2$, the ring of rational integers, we conclude that $G$ is subnormal in $U(Z G)$ if, and only if, $G$ is an abelian or a Hamiltonian 2-group; as a corollary we obtain [8], Theorem 1 .

Our technique, which already appears in [3], is inspired by Herstein

Received 2 November 1984. This work was completed while the author was visiting the University of Alberta, and was supported by CNPq.

Copyright Clearance Centre, Inc. Serial-fee code: 0004-9727/85 $\$$ A2.00 +0.00 . 
[5]. We are indebted to Arnaldo Mandel for many useful conversations.

\section{Preliminary results}

If $H$ is a subgroup of $G$ we write $H \triangleleft G$ to indicate that $H$ is normal in $G$, and $H \triangleleft \triangleleft G$ to indicate that $H$ is subnormal in $G$.

LEMMA 2.1. Let $\Pi$ be a nonempty subset of the set of rational primes, and let $N$ be a I-subgroup of $G, N \triangleleft \triangleleft G$. Then there exists a I-subgroup $M, M \triangleleft G$, such that $N \subseteq M$.

Proof. See [5], Lemma 1.

Let $K G[X]$ be the polynomial ring in the commutative indeterminate $X$ with coefficients in $K G$.

LEMMA 2.2 (van der Monde determinant argument). Let $f(X)$ be an element of $K G[X]$. If $f(X)$ assumes the same value for infinitely many elements of $K$, then $f$ is constant.

Proof. The claim is obviously equivalent to the statement that, if $f(X)$ has an infinite number of zeros in $K$, then $f(X)$ is zero. Hence, let $f(X)=a_{0}+a_{1} X+\ldots+a_{n} X^{n}$ and let $\lambda_{0}, \lambda_{1}, \ldots, \lambda_{n}$ be a set of $n+1$ distinct zeros of $f(X)$ in $K$. Then, using matrix notation

$$
\left[a_{0}, a_{1}, \ldots, a_{n}\right]\left[\begin{array}{cccc}
1 & 1 & \ldots & 1 \\
\lambda_{0} & \lambda_{1} & \ldots & \lambda_{n} \\
\lambda_{0}^{2} & \lambda_{1}^{2} & \ldots & \lambda_{n}^{2} \\
\vdots & & & \\
\lambda_{0}^{n} & \lambda_{1}^{n} & \ldots & \lambda_{n}^{n}
\end{array}\right]=[0,0, \ldots, 0] .
$$

The matrix is invertible, whence $\left[a_{0}, a_{1}, \ldots, a_{n}\right]=[0,0, \ldots, 0]$.

PROPOSITION 2.3. Let $m$ and $n$ be positive integers, $n>1$, let $f(X)=a_{0}+a_{1} X+\ldots+a_{m n} X^{m n}$ be an element of $K G[X]$, and suppose that the rational function $g(X)=f(X) /\left(1-X^{n}\right)^{m}$ assumes the same value for infinitely many elements of $K$. Then $a_{1}=0$.

Proof. Let $B$ be an infinite subset of $K$, and let $c$ be an 
element of $K G$ such that

$$
g(\lambda)=c \text { for every } \lambda \in B \text {. }
$$

Then $f(\lambda)=c\left(1-\lambda^{n}\right)^{m}$ for every $\lambda \in B$, and since the right hand side has no term of first degree in $\lambda$, by Lemma 2.2, $a_{1}=0$.

Given an element $u \in K G$, char $K=p>0$, we define an inner derivation in $K G$ in the usual way: if $w \in K G$, then $w^{(0)}=w$, $w^{\prime}=w u-2 a v$ and $w^{(i+1)}=\left(w^{(i)}\right)^{\prime}$, for every $i \geq 1$. Now, if $r$ is a positive integer, we recall the formula

$$
\omega^{\left(p^{r}\right)}=w u^{p^{r}}-u^{p^{r}}
$$

\section{Normal subgroups of the group of units}

We denote by $\zeta U(K G)$ the center of $U(K G)$.

THEOREM 3.1. Let $K$ be an infinite field, let $G$ be a group generated by torsion elements and let $H$ be a subgroup of $U(K G)$. Suppose moreover that, either $H$ is finite or $H$ is abelian and $H \subseteq G$. Then $H \diamond U(K G)$ if, and only if, $H \subseteq \zeta U(K G)$.

Proof. Only necessity requires a proof.

(i) $H$ is finite. Let $h \in H$, and let $g \in G$ be a generator of $G$ of order $n$. Let $\lambda$ be an element of $K$ such that $\lambda^{n} \neq 1$. Then

$$
(1-\lambda g)^{-1}=\frac{1+\lambda g+\ldots+\lambda^{n-1} g^{n-1}}{1-\lambda^{n}},
$$

and let us consider for such $\lambda$ the element

$$
h_{\lambda}=h(1-\lambda g) h^{-1}(1-\lambda g)^{-1} \text {. }
$$

Developing the above expression as a polynomial in $\lambda$ we obtain

$$
h_{\lambda}=\frac{1+\lambda\left(g-h g h^{-1}\right)+\lambda^{2} q_{2}(g, h)+\ldots+\lambda^{n} q_{n}(g, h)}{1-\lambda^{n}}
$$

where $q_{2}(g, h), \ldots, q_{n}(g, h) \in K G$. 
Now, since $H \triangleleft U(K G)$, the rational function

$$
\phi(X)=\frac{1+\left(g-h g h^{-1}\right) x+q_{2} x^{2}+\ldots+q_{n} x^{n}}{1-X^{n}}
$$

assume values in $H$, for an infinite number of $\lambda$ in $K$. Thus, since $H$ is finite, the Pigeon-Hole Principle implies that $\phi(X)$ assumes the same value for infinitely many $\lambda$ in $K$. By Proposition 2.3,

$$
g-h g h^{-1}=0
$$

and

$$
g h=h g .
$$

Since every $h \in H$ commutes with every torsion generator $g \in G$ the conclusion follows.

(ii) $H$ is abelian and $H \subseteq G$. Arguing as in (i), we observe that for infinitely many $\lambda$ belonging to $K$ we have that $\left(1-\lambda^{n}\right) \phi(\lambda) \in K H$. Hence, solving the system of equations as in Lemma 2.2 , we conclude that

$$
g-h g h^{-1} \in K H
$$

or

$$
g h-h g \in K H \text {. }
$$

So, if $g \in G \backslash H$ is a torsion generator of $G$, we obtain $g h=h g$, as was to be proved.

\section{Subnormal subgroups of the groups of units}

We cannot handle the subnormality question as easily, and so we will study separately the cases $p=0$ and $p>0$.

Let $R$ be an integral domain. We denote by $V(R G)$ the group of normalized units of $R G$, that is, the set of elements of $U(R G)$ with augmentation one. The proposition below is an easy generalization of [9], Theorem II 5.1 .

PROPOSITION 4.1. Let $G$ be a group, let $R$ be an integral domain of characteristic 0 such that no rational prime is a unit of $R$, and let $H$ be a torsion subnormal subgroup of $V(R G)$. Then $H \subseteq G$ and $H$ is an 
abelican or a Hamiltonian 2-group, with every subgroup normal in $G$.

Proof. By Lemma 2.1 there exists a torsion normal subgroup $N$ of $V(R G)$ such that $H \subseteq N$. By [9], Theorem II 5.1, the conclusion follows.

THEOREM 4.2. Let $G$ be a torsion group. Then $G \triangleleft \triangleleft U(2 G)$ if, and only if $G$ is an abelian or a Hamiltonian 2-group.

Proof. Necessity. Since $G \subseteq V(Z G)$ we have that $G \triangleleft \triangleleft V(Z G)$, and applying Proposition 4.1 we arrive at the desired conclusion.

Sufficiency. Apply [9], Corollary II 2.5.

The corollary below implies in particular, [8], Theorem 1 .

COROLLARY 4.3. Let $G$ be a torsion group. Then $U(Z G)$ is nilpotent if, and only if, $G$ is an abelian or a Hamiltonian 2-group.

Proof. Necessity. Since $U(Z G)$ is nilpotent every subgroup of $U(Z G)$ is subnormal. So $G \triangleleft \triangleleft U(Z G)$, and by Theorem 4.2, $G$ is an abelian or a Hamiltonian 2-group.

Sufficiency. If $G$ is abelian there is nothing to prove. If $G$ is a Hamiltonian 2-group apply [9], Corollary II 2.5.

THEOREM 4.4. Let $K$ be a field of characteristic 0 , let $G$ be a torsion group and let $H$ be a subgroup of $G$. Then $H \triangleleft \Delta U(K G)$ if, and only if $H \subseteq \zeta G$.

Proof. Only necessity requires a proof.

Since $H \triangleleft \triangleleft U(K G)$ it follows that $H \Delta \triangleleft U(Z G)$ so, by Proposition 4.1, $H$ is either an abelian or a Hamiltonian 2-group.

Suppose that $H$ is a Hamiltonian 2-group. Then $H=K_{8} \times E$, the direct product of the quaternion group of order 8 by an elementary abelian 2-group $E$. So $K_{8} \triangleleft H$, and $K_{8} \triangleleft \triangleleft U(K G)$ implies that $K_{8} \triangleleft \triangleleft U\left(K K_{8}\right)$, in contradiction to [2], Theorem 2.4 .

Therefore $H$ is abelian and we claim that $H$ is central. Suppose not. Then there exist $a \in H$ and $g \in G$ such that $(a, g) \neq 1$, and since $\langle a\rangle \triangleleft G$ it follows that $\tilde{G}=(a, g)$, the subgroup generated by $a$ and $g$, is finite. Again $\langle a\rangle \triangleleft H \triangleleft \triangleleft U(K G)$ and so $(a\rangle \triangleleft \triangleleft U(K \tilde{G})$, in contradiction to [2], Theorem 2.4 . 
Now we turn our attention to the case $p>0$.

PROPOSITION 4.5. Let $K$ be an infinite field of characteristic $p>0$, let $G$ be a group generated by torsion elements, and let $H$ be $a$ subnormal subgroup of $U(K G)$ such that either $H$ is finite or $H$ is nizpotent. Then there exists a positive integer $l \geq 1$ such that $H^{2} \subseteq \zeta U(K G)$

Proof. (i) $H$ is finite. Let $H=N_{r} \triangleleft N_{r-1} \triangleleft \ldots \triangleleft N_{1} \triangleleft N_{0}=U(K G)$ be a subnormal series for $H$, let $h \in H$, let $g \in G$ be a generator of $G$ of order $n$, and let $\lambda \in K$ be such that $\lambda^{n} \neq 1$. Then

$$
(1-\lambda g)^{-1}=\frac{1+\lambda g+\ldots+\lambda^{n-1} g^{n-1}}{1-\lambda^{n}}
$$

and we define recursively,

$$
c_{\lambda I}=(h,(1-\lambda g)), c_{\lambda 2}=\left(c_{\lambda I}, h\right), \ldots, c_{\lambda(i+1)}=\left(c_{\lambda i}, h\right)
$$

for every positive integer $i$, and where $(x, y)=x y x^{-1} y^{-1}$.

As before

$$
c_{\lambda I}=\frac{1+\lambda\left(g^{\prime} h^{-1}\right)+\lambda^{2} q_{2}(g, h)+\ldots+\lambda^{n} q_{n}(g, h)}{1-\lambda^{n}}
$$

with $q_{2}, \ldots, q_{n} \in K G$. In general, an easy induction argument shows that

$$
c_{\lambda m}=\frac{1+\lambda\left(g^{(m)} h^{-m}\right)+\lambda^{2} s_{2}(g, h)+\ldots+\lambda^{m m} s_{m n}(g, h)}{\left(1-\lambda^{n}\right)^{m}}
$$

where $s_{2}, \ldots, s_{m n} \in K G$.

Now choose a positive integer 2 such that $m=p^{2}>r$. Then $c_{\lambda m} \in H$ for every $\lambda \in K$ such that $\lambda^{n} \neq 1$, and since $H$ is finite the Pigeon-Hole Principle implies that the rational function

$$
\phi(X)=\frac{1+X\left(g^{(m)} h^{-m}\right)+X^{2} s_{2}+\ldots+X^{m n} s_{m n}}{\left(1-X^{n}\right)^{m}}
$$


assumes the same value for infinitely many $\lambda \in K$. By Proposition 2.3,

$$
g^{(m)} h^{-m}=0
$$

and by formula (I) we have

$$
\begin{aligned}
& g\left(p^{2}\right)=g h^{2}-h p^{2} g=0, \\
& g h^{2}=h p^{2} g,
\end{aligned}
$$

and the conclusion follows.

(ii) $H$ is nilpotent. As in (i), we define inductively the elements $c_{\lambda i}$ for every positive integer $i$. Since $H$ is nilpotent there exists a positive integer 2 such that, for $m=p^{2}$, we have $c_{\lambda m}=1$ for every $\lambda \in K$ with $\lambda^{n} \neq 1$. Now repeat the argument of $(i)$.

THEOREM 4.6. Let $K$ be an infinite field of characteristic $p>0$, let $G$ be a group generated by torsion elements, and let $H$ be a subgroup of $U(K G)$ such that either $H$ is finite or $H$ is torsion nilpotent. Then $H \triangleleft \triangleleft U(K G)$ if, and only if:

(a) $H=P \times Q$, the direct product of a p-group $P$ by $a$ $p^{\prime}$-group $Q$;

(b) there exists a positive integer 2 such that

$$
P^{2} \times Q \subseteq \zeta U(K G) \text { and } P \triangleleft \triangleleft U(K G) \text {. }
$$

Proof. Necessity. (i) $H$ is finite. By Proposition 4.5 there exists a positive integer $l$ such that

$$
{ }_{H}^{p^{2}} \subseteq \zeta U(K G) \cap H \subseteq \zeta H
$$

Therefore $H / \zeta H$ is a finite $p$-group and hence $H$ is nilpotent. Thus we can write $H=P \times Q$, the direct product of a finite $p$-group $P$ by a finite $p^{\prime}$-group $Q$. Moreover, since the order of every element of $Q$ is prime to $p$ we have that $Q \subseteq \zeta U(K G)$.

Now, since $P \triangleleft H$, it follows that $P \triangleleft \triangleleft U(K G)$.

(ii) $H$ is torsion nilpotent. Once more, since $H$ is torsion 
nilpotent, we can write $H=P \times Q$, the direct product of a $p$-subgroup $P$ by a $p^{\prime}$-subgroup $Q$ and repeat the reasoning above, invoking Proposition 4.5.

Sufficiency. Since $P \triangleleft \triangleleft U(K G)$ and $Q \subseteq \zeta U(K G)$ it follows that $\ddot{H}=P \times Q \triangleleft \triangleleft U(K G)$.

As a consequence of Theorem 4.6 above we can obtain the result of Pearson and Taylor [7] for infinite fields of nonzero characteristic.

COROLLARY 4.7. Let $K$ be an infinite field of nonzero characteristic $p$ and let $G$ be a finite group. Then a subgroup $H$ of $G$ is subnormal in $U(K G)$ if, and only if, $H=P \times Q$ where $P$ is contained in $O_{p}(G)$, the maximum normal $p$-subgroup of $G$, and $Q$ is a $p^{\prime}$-group contained in $\zeta G$.

Proof. Necessity. By Theorem 4.6, $H=P \times Q$, with $P$ a subnormal p-subgroup of $U(K G)$ and $Q \subseteq \zeta U(K G)$ a $p^{\prime}$-subgroup. But $P \subseteq G$, and since $P \triangleleft \triangleleft U(K G)$ implies $P \triangleleft \triangleleft G$, by Lemma 2.1, $P \subseteq O_{p}(G)$. The remaining part follows from the fact that $\zeta U(K G) \cap G=\zeta G$.

Sufficiency. See [7].

COROLLARY 4.8. Let $G$ be a finite group and let $K$ be an infinite field. Then $G \triangleleft \triangleleft U(K G)$ if and only if $U(K G)$ is nilpotent.

Proof. If $U(K G)$ is nilpotent certainly $G \triangleleft \triangleleft U(K G)$. So, let us assume that $G \triangleleft \triangleleft U(K G)$.

If char $K=0$, by Theorem 4.4, $G$ is abelian, so $U(K G)$ is nilpotent.

If $\operatorname{char} K=p>0$, by Theorem 4.6, $G=P \times Q$, the direct product of a $p$-subgroup $P$ by a central $p^{\prime}$-subgroup $Q$. Now by [1], $U(K G)$ is nilpotent.

COROLLARY 4.9. Let $K$ be an infinite field of characteristic $p>0$ and let $G$ be a group generated by torsion elements. Then a subnormal $p^{\prime}$-subgroup $H$ of $U(K G)$ is nilpotent if, and only if, $H \subseteq \zeta U(K G)$.

COROLLARY 4.10. Let $K$ be an infinite field of characteristic $p>0$, and let $G$ be a torsion solvable group without p-elements. Then $G \triangleleft \triangleleft U(K G)$ if, and only if $G$ is abelian. 
Proof. Suppose that $G \triangleleft \triangleleft U(K G)$. By [4], Lemma 1, G contains a nilpotent characteristic subgroup $N$ of class at most two, such that $N \supseteq C_{G}(N)$, the centralizer of $N$ in $G$.

Since $N$ is a nilpotent $p^{\prime}$-subgroup with $N \triangleleft \triangleleft U(K G)$, by Corollary 4.9 it follows that $N \subseteq \zeta G$. So, from $N \supseteq C_{G}(N)$ we conclude that $G=N$ and $G$ is abelian.

\section{References}

[1] J.M. Bateman and D.B. Coleman, "Group algebras with nilpotent unit groups", Proc. Amer. Math. Soc. 19 (1968), $448-449$.

[2] J.Z. Goncalves, "Free groups in subnormal subgroups and the residual nilpotence of the group of units of group rings", Comad. Math. BuzL. 27 (1984), 365-370.

[3] J.Z. Goncalves and A. Mandel, "Are there free groups in division rings?", Israel J. Math. (to appear).

[4] B. Hartley, "Finite groups of automorphisms of locally soluble groups", J. Algebra 57 (1979), 242-257.

[5] I.N. Herstein, "Multiplicative commutators in division rings, II", Rend. Circ. Mat. Palermo (2) 29 (1980), 485-489.

[6] K.R. Pearson, "On the units of a modular group ring II", BuzZ. Austral. Math. Soc. 8 (1973), 435-442.

[7] K.R. Pearson and D.E. Taylor, "Groups subnormal in the units of their modular group ring", Proc. London Math. Soc. (3) 33 (1976), 313-328.

[8] C. Polcino Milies, "Integral group rings with nilpotent unit groups", Canad. J. Math. 28 (1976), 954-960.

[9] S.K. Sehgal, Topics in group rings (Marcel Dekker, New York, 1978).

Instituto de Mat e Estatistica,

Universidade de Sao Paulo,

Cx Postal 20570 Ag Iguatemi,

ol000 Sao Paulo, Brasil. 